\title{
MedienPädagogik
}

Zeitschrift für Theorie und Praxis der Medienbildung

\section{Inszenierungsmöglichkeiten eines mediengestützten Sportunterrichts}

\author{
Mareike Thumel, Anja Schwedler-Diesener, Steffen Greve, Jessica
} Süßenbach, Florian Jastrow und Claus Krieger

\section{Zusammenfassung}

Medienbildung stellt eine Querschnittaufgabe aller Schulfächer, so auch des Sportunterrichts dar, die in ihrer unterrichtlichen Umsetzung auch die jeweiligen fachdidaktischen und grundschulspezifischen Ansprüche erfüllen soll. Dies kann eine Herausforderung für die Unterrichtsfächer bzw. die unterrichtenden Fachlehrkräfte sein. Zur Integration dieser medienbildungsbezogener Themen in die konkrete (Fach-)Unterrichtspraxis liegen mittlerweile zwar vereinzelt Beispiele, jedoch für den hier fokussierten Sportunterricht noch keine systematische Entwicklung und Evaluation entsprechender Unterrichtsvorhaben vor. Im Rahmen eines kooperativen Explorationsprojektes wurden entsprechende Versuche unternommen, einen digital gestützten Grundschulsportunterricht zu planen, durchzuführen und zu reflektieren. Dieser Beitrag setzt sich mit der Reflexion der Unterrichtseinheiten vor dem Hintergrund einer pädagogischen Beschäftigung mit den Begriffen Innovation, Mobilität und Digitalität auseinander und fragt in diesem Zusammenhang nach Möglichkeiten der Inszenierung eines mediengestützten und medienbildenden Sportunterrichts der Grundschule. 


\title{
Ways of staging a media-supported physical education classroom
}

\begin{abstract}
Media education is a cross-sectional task of all school subjects, including physical education (PE). When implemented into teaching, it should meet the respective subject-specific and level-specific requirements, for example those of primary school education. This poses a major challenge for the subjects or rather the subject teachers. By now, there are a few examples of how these interdisciplinary educational topics can be integrated into specific (subjectrelated) teaching practice, but suitable teaching projects for $P E$, our focus, have not been developed and evaluated yet. Within a cooperative exploration project, we started attempts to this end, which are outlined in this article. These attempts are then reflected focussing on the compatibility of differing requirements with regard to their possibilities and limitations.
\end{abstract}

\section{Einführung: Innovativ, mobil und digital! Bildungs- politische Forderungen und ihre Begegnung mit dem Pädagogischen im Grundschulsportunterricht}

Innovativ, mobil und digital - diese Eigenschaften beschreiben nicht nur im bildungspolitischen Kontext, sondern auch in der gesellschaftlichen Breite einen anzustrebenden modernen Schulunterricht. Doch was verbirgt sich genau hinter diesen Eigenschaften? Was sind Beweggründe für eine Unterrichtsentwicklung in diese Richtung? Wie können Innovation, Mobilität und Digitalität Eingang in den (Grund-)Schulunterricht finden? Was bedeutet diese Entwicklung für die Inszenierung (vgl. Dietrich und Landau 1990) des Sportunterrichts?

In welche gesellschaftlichen oder wirtschaftlichen Bereiche man auch schaut, die Menschen beschäftigen sich mit dem durch Medienentwicklungen geprägten Wandel ihres privaten und beruflichen Lebensumfeldes. So ist es nicht verwunderlich, dass auch aus bildungspolitischer Richtung die Digitalisierung aktuell einer der zentralen Ankerpunkte für den Anstoss weitreichender Schul- und Unterrichtsentwicklungsprozesse darstellt. 
Während Innovation, Mobilität und Digitalität allen voran in der Wirtschaft an die Hoffnung auf mehr Sichtbarkeit, eine schnelle Effizienzsteigerung und damit höhere Gewinne gekoppelt sind, können diese Ansprüche nur bedingt im Bildungsbereich Geltung haben. Sie brauchen hier ein gesondertes Bedeutungsspektrum und in Abgrenzung an wirtschaftlichen Interessen orientierten Wert. Mit Blick auf das Primat des Pädagogischen, welches sowohl in dem Beschluss der $\mathrm{KMK}$ «Bildung in der digitalen Welt» als auch in der Strategie des BMBF betont wird sollen Innovation, Mobilität und Digitalität somit nicht in ihrer wirtschaftlich geprägten Bedeutung auf den Kontext von Schule übertragen werden. Kammerl (2018) stellt heraus, dass sowohl die Entwicklungsphasen der Heranwachsenden Berücksichtigung finden müssen, als auch der Zusammenhang zwischen Werten, Zielen und Erziehungsmassnahmen (Kammerl 2018, 22).

Um eine bildungsorientierte Schul- und Unterrichtsentwicklung zu evozieren, werden im Folgenden Überlegungen zu Neuerungen auch aus dieser Richtung betrachtet dargelegt. Im Anschluss werden Möglichkeiten dargelegt, wie Digitalität, Mobilität und Innovation Eingang in den Grundschulunterricht finden können. Dies wird durch medienpädagogischen Überlegungen zum Lernen mit und über Medien gerahmt und durch bildungpolitische Bemühungen ergänzt. Um diese Gedanken an konkreten Beispielen aus dem Projekt «Mein Sport» der Autorinnen und Autoren zu verdeutlichen, werden die Grundzüge des sportdidaktischen Diskurs des Grundschulsportunterrichts dargelegt. Im dritten Kapitel wird das MeInSport - Projekt und daraus vier Unterrichtsbeispiele skizziert, die unterschiedliche Einsatzmöglichkeiten von Tablets im Sportunterricht von Erstund Zweitklässlern aufzeigen um dann im abschliessendenbeschliessenden Kapitel an diesen Beispielen über Inszenierungsmöglichkeiten eines mediengestützen und medienbildenden Sportunterrichts zu reflektieren.

\subsection{Innovation}

Innovation beschreibt im Allgemeinen die Neuerung oder Erneuerung einer Sache. In diesem Bedeutungszusammenhang wird oftmals etwas Kreatives, Schöpferisches, Fortschrittliches, Zukunftsweisendes oder Originelles assoziiert. Das Innovieren ist eine der vier Kompetenzbereiche, 
welche die Kultusministerkonferenz in ihrem Beschluss von 2019 (Kultusministerkonferenz 2019; Kultusministerkonferenz KMK 2012) Standards für die Lehrerbildung: Bildungswissenschaften in überarbeiteter Form festgeschrieben hat. Der Kompetenzbereich gliedert sich in drei Kompetenzen: «Lehrkräfte sind sich der besonderen Anforderungen des Lehrerberufs bewusst und beziehen gesellschaftliche, kulturelle und technologische Entwicklungen in ihr Handeln ein. [...] Lehrkräfte verstehen ihren Beruf als ständige Lernaufgabe und entwickeln ihre Kompetenzen weiter. [...] Lehrkräfte beteiligen sich an der Schul- und Unterrichtsentwicklung» (Kultusministerkonferenz 2019).

Innovation spiegelt sich hier auf zwei Ebenen, einerseits in der Innovation der eigenen Rolle als Lehrkraft und andererseits in der Innovation des Arbeitsumfeldes, also der Schule und des Unterrichts. Innovation wird in der Kompetenzbeschreibung jedoch weniger als originelle Erschaffung oder kreative Schöpfung von etwas Neuem gesehen. Vielmehr sind die Reflexion und die Entwicklung von bestehenden Strukturen und Zuständen oder der Transfer von einer Situation in eine andere und die Neukombination von Theoretischem und Praktischem hier als zentrale Merkmale des Innovierens zu verstehen.

Innovationen jeglicher Art brauchen im pädagogischen Sinne ein klares Ziel, nämlich die Verbesserung unterrichtlicher und schulischer Bildungsbedingungen für Schülerinnen und Schüler. Diese ist mit Sicherheit durch Reflexion, Transfer und Neukombination zu erwirken, doch könnte auch die erste Lesart, welche Kreativität und Neuerung in sich trägt, stärker in den Vordergrund rücken. Beispielsweise zeigt das SAMR-Modell ${ }^{1}$ (vgl. Puentedura 2006) das Innovationspotenzial von Aufgabenformaten durch den Einsatz von Tablets im Unterricht in vier Stufen (Substitution, Augmentation, Modification und Redefinition). Die beiden erstgenannten unteren Stufen, übersetzt mit Ersetzung und Erweiterung, stehen für die Art und Weise der Medienintegration, welche lediglich mit anderen Unterrichtswerkzeugen gegebenenfalls mit funktionalen Verbesserungen

1 Das Modell vernachlässigt jedoch die Frage nach dem Ziel der Innovationspotenziale. Es werden weder pädagogische noch didaktische oder fachdidaktische Leitideen der Unterrichtsentwicklungen berücksichtigt, sodass das Modell zu einer technikzentrierten Sichtweise von Innovation neigt. 
einhergehen. Die beiden oberen Stufen, Modifikation und Neubestimmung, beschreiben einen Tableteinsatz, der zu einer Neugestaltung von Lernaufgaben beziehungsweise zur Entwicklung sonst undenkbarer Aufgaben führt (Bastian 2017).

\subsection{Mobilität}

Mobilität steht grundlegend für Beweglichkeit, also ganz allgemein das Verändern einer Position eines Lebewesens oder eines Gegenstandes im Raum. Im Kontext von Schule kann Mobilität unter anderem als Ausgangspunkt der Schulraumgestaltung, als die Betrachtung von Bewegungsabläufen im Unterricht, als zentrales Ziel des Sportunterrichts, als soziologisches Unterrichtsthema oder im Sinne einer gedanklichen Mobilität der schulischen Beteiligten betrachtet werden. Insbesondere die ersten beiden Punkte bieten an dieser Stelle mehrere Anlässe zu weiteren Überlegungen: Das gedankliche Bild des Schulunterrichts ist seit jeher geprägt durch eine Anordnung von Tischen und Stühlen, einem Lehrkräftepult und einer Tafel. Die Abbilder und Vorstellungen dazu sind über Jahrzehnte hinweg überaus starr, so wie auch die Beweglichkeit von Personen und einzelner Elemente im Schulunterricht oftmals eher als Ausnahmesituation oder Störfaktoren (heimliches Weiterreichen von Briefen, Radiergummi werfen, durch den Raum laufen, mit dem Stuhl kippeln etc.) angesehen wird.

In Konzepten zur Unterrichtsentwicklung kann Mobilität aber durchaus auch als Möglichkeit und Chance für das Lernen wahrgenommen werden. Die Blickrichtung muss dafür geweitet werden, um mehr die Potentiale als die Fallstricke zu sehen. Mobilität als Chance zu begreifen, bedeutet Möglichkeiten zu schaffen, sodass Schülerinnen und Schüler ihre Lernwege im Unterricht räumlich selbstständig und eigenverantwortlich (bzw. mit Unterstützung der Lehrkraft) organisieren (z.B. bei einer Stationsarbeit) oder die Anordnung von Möbeln, Medien und Materialien der Unterrichtssituation nach Bedarf angepasst werden kann. Ein ganz anderes, aber ebenso starres Bild kursiert für den Sportunterricht. Hier steht die Bewegung im Vordergrund. Der Einsatz von digitalen Medien scheint auf den ersten Blick unzweckmässig, da die Bewegungszeit leiden könnte (Wendeborn 2019, 12). Hier können aber gerade durch mobile Medien, 
wie Tablets auch in Sporthallen Sachverhalte visualisiert und dargestellt werden und auch Bewegungen der Schülerinnen und Schüler durch das Filmen in der Sporthalle im Klassenzimmer präsentiert werden, sodass die Bewegungslernzeit an Qualität gewinnt (vgl. Kapitel 3). Unter Einbezug psychologischer, philosophischer, soziologischer und natürlich auch pädagogischer Forschungsbefunde und Kriterien der Schulraumgestaltung (u.a. nachzulesen bei Kahlert, Nitsche, und Zierer 2013), sollte der Mobilität insofern ein pädagogischer Wert beigemessen werden, als dass sie die Beweglichkeit von Menschen, Dingen, Gedanken, Gefühlen oder Wissen heraushebt und beim Nachdenken über Schule und Unterricht zum Thema macht.

\subsection{Digitalität}

Digitalität basiert auf zwei Zeichen, o und 1. Es ist ein binäres System, welches Möglichkeit bietet digitale Texte, Bilder, Videos und Töne zu erzeugen, zu speichern und zu übertragen (Döbeli Honegger 2016). Digitalität kann auf Grundlage dieser Definition zunächst nur bedingt im Licht von Schule und Unterricht betrachtet werden, ohne gleichzeitig die entgegengesetzte Blickrichtung (Schule und Unterricht im Lichte der Digitalität) einzunehmen. Die pädagogische Bedeutung von Digitalität hat so gesehen viele Gesichter und Bezeichnungen: Sie taucht unter anderem in Fragen der unterrichtlichen Lehrmittelwahl (Petko, Döbeli Honegger, und Prasse $2018,166)$ auf, in denen die Autorin und Autoren die Möglichkeit durch die Gestaltung von problemlösenden und komplexen Unterrichtsformate zur Bewältigung des digitalen Wandels beizutragen sehen.

Des Weiteren werden schulische Organisation und Kommunikation medienvermittelt geführt und dies beinhaltet nicht nur die administrativ-organisatorischen Prozese, sondern auch Lern- und Lehrprozesse, Urheberrechtliche Unwägbarkeiten oder örtlichen, zeitlichen und sozialen Entgrenzungsphänomenen (Herzig und Aßmann 2014) werden gesehen, indem digitaler Medien ortsunanhängig und von der physischen Anwesenheit sowie von verbindlichen Zeitfenstern unanhängig für Lernprozzese eingesetzt werden können und auch das informell erworbene Wissen durch die ausserschulische Mediennutzung wirken sich auf den 
Unterricht und schulische Lehr-Lernprozesse aus (ebd.). Darüber hinaus steht Digitalität bzw. der verwandte Begriff der Digitalisierung oftmals in engem Zusammenhang mit veränderungsverheissenden Reflexionsanlässen grundlegender Gegebenheiten von Schule und Unterricht: Lernen sei neu zu definieren (vgl. Dräger und Müller-Eiselt 2018) und das System Schule neu zu denken (Jörissen und Münte-Goussar 2015) beziehungsweise in Gänze zu hinterfragen (vgl. Döbeli Honegger 2016, 41). Hinzu summieren sich Forderungen nach neuen Lehrplänen und neuen Inhalten, welche sich unter anderem dem Ziel einer medienpädagogischen und informatikdidaktischen Kompetenzförderung (Rummler et al. 2016) verschreiben.

Als Trio können Innovation, Mobilität und Digitalität in Überlegungen zu einer pädagogisch orientierten Unterrichtsentwicklung viele Potenziale freilegen. Im Folgenden soll das Lernen mit und über Medien aus medienpädagogischer und sportdidaktischer Perspektive erläutert und dann auf den Sportunterricht in der Grundschule eingegangen werden

\section{Zielvorstellungen der Unterrichtsentwicklung im Grundschulsportunterricht}

\subsection{Die mediatisierte Grundschule}

Wie können Digitalität, Mobilität und Innovation Eingang in den Grundschulunterricht finden? Diese Frage kann zunächst mit Zahlen und Studienergebnissen in Bezug auf die Medienausstattung der Grundschulen, Nutzungsformen und -häufigkeiten beantwortet werden.

Seit 1999 werden alle zwei Jahre quantitative Daten über den Stellenwert von Medien im Alltag der sechs bis 13-jährigen durch die KIM-Studie erhoben und ausgewertet. Hier wird auch nach der Mediennutzung in der Schule gefragt, so geben $31 \%$ der Kinder an zumindest einmal pro Woche den Computer zu nutzen (Mpfs. Medienpädagogischer Forschungsverbund Südwest 2019). 83,9\% der Kinder besuchen Schulen mit Computerräumen und 60\% der Kinder erhalten Zugang zu den Geräten unmittelbar im Klassenraum oder angrenzenden Arbeitsräumen (Eickelmann et al. 2014). 
Seltener ist die Nutzung mobiler Geräte wie Tablets (8\%), Laptops (15\%) und das eigene Smartphone (16\%) im Unterricht (vgl. Mpfs 2019, 50f).

Auf Grund des Klassenlehrerprinzips werden gerade in Grundschulen die Unterrichtsgestaltung und die Medienintegration meist von der einzelnen Lehrkraft entscheidend geprägt. Hat die Klassenlehrerin bzw. der Klassenlehrer grundsätzlich Vorbehalte digitalen Medien gegenüber, «kann ein Grundschulkind die Grundschule durchlaufen, ohne jemals digitale Medien zum Lernen genutzt zu haben» (Eickelmann und Vennemann 2014, 82). Die meisten Lehrkräfte weisen diesbezüglich eine positive Haltung auf, sodass etwa drei Viertel aller Grundschulkinder von Lehrkräften unterrichtet werden, die nach Eigenauskunft Computer in ihren Unterricht integrieren. Konkrete Tätigkeiten sind, der KIM-Studie 2018 zufolge, in erster Linie das Verfassen von Texten, was jedes dritte Kind etwa ein Mal pro Woche macht. Etwa 24\% nutzen das Internet zur Recherche, 23\% verwenden Lernprogramme. Produktive Nutzungsformen wie zum Beispiel die Bildbearbeitung konnten bei 11\% der Schülerinnen und Schüler festgestellt werden (vgl. Mpfs 2019, 51). Für den Sportunterricht können keine expliziten Aussagen getroffen werden.

Einen weiteren Eingang in die Schule finden digitale Medien über die ausserschulischen Erfahrungen der Kinder. Die Bertelsmann Stiftung stellt in ihrer qualitativen Studie in Gruppengesprächen mit Grundschulkindern fest, dass das private Umfeld von vielerlei Medien bestückt ist und die Kinder viele ausserschulische Medienerfahrungen mitbringen. Gleichzeitig berichteten alle Kinder von Verboten, welche die privaten Geräte aus dem Schulalltag ausschliessen. Es wird für Konzepte plädiert, «die ein integriertes digitales Lernen zu Hause und in der Schule ermöglichen» (Thom et al. 2017). Aus Richtung der Grundschulpädagogik befand eine Studie, welche die Erfahrungen eines tabletbasierten Unterrichts aus der Perspektive von Grundschulkindern nachzeichnen möchte, Aspekte der Selbstund Fremdkontrolle bei der Gerätenutzung und das Ineinanderübergehen von Spielen und Lernen als besonders relevante Ankerpunkte der Unterrichtsgestaltung (Zimmermann 2017). Auch berichten Alexander Tillmann und Claudia Bremer in ihren Ergebnissen einer Studie zum Tableteinsatz im Grundschulunterricht, 
«dass die Tablets mehr und mehr als Werkzeug zur Gestaltung und Unterstützung von kreativen Lernprozessen zum Einsatz kamen und Handlungsfreiheit und Kontrolle vermehrt an Schülerinnen und Schüler abgegeben wurde» (Tillmann und Bremer 2017, 261),

obwohl die Lehrkräfte diesem Schritt anfangs skeptisch gegenüberstanden. Die didaktisch-methodische Verschiebung von einer inhaltsorientierten Vermittlungsfunktion hin zu prozess- und gestaltungsorientiertem Tableteinsatz, führte zu einer besseren Zentrierung des Unterrichts auf die Schülerinnen und Schüler, welche sich durch die «Entwicklung intrinsischer Motivation und dem Zusammenhang zwischen der Entwicklung von Interesse, wahrgenommenem Vergnügen und Wahlfreiheit» (ebd.) äussert.

Als die Schule für alle, steht die Grundschule vor der Herausforderung, Kinder mit heterogenen Voraussetzungen in Lerngruppen zu unterrichten. Digitale und mobile Medien können die Lehrkräfte dabei unterstützen, werden aber im Rahmen schulischer Medienbildung auch zum Unterrichtsgegenstand. Den Medien kommen demnach im Unterricht verschiedene Funktionen und Rollen zu, welche einer fachdidaktischen und medienpädagogischen Verortung bedürfen.

\subsection{Das Lernen mit Medien}

Digitale Medien wie Computer, Tablets oder Smartphones und digitale Medienangebote wie das Internet, Datenbanken, Computerprogramme oder Apps können Hilfsmittel und Werkzeuge für das unterrichtliche beziehungsweise fachliche Lernen darstellen ${ }^{2}$. Es werden ihnen zahlreiche Potenziale zugesprochen und gleichzeitig allerhand Fallstricke angenommen ${ }^{3}$ : Der Einsatz von Medien und Medienangeboten erhöhe einerseits die Methodenvielfalt, ermögliche neue Kommunikations- und

2 Fachdidaktische und Medienpädagogische Ideen für den tabletgestützten Unterricht sind Krauthausen et al. (2020) oder Junge und Niesyto (2019) zu finden.

3 Eine Aufzählung von Argumenten für und gegen digitale Medien als Werkzeug und Thema in Schule und Unterricht findet sich von Beat Döbeli Honegger (2016). 
Kollaborationswege, biete ansprechende Visualisierungs- und Simulationsoptionen, sei motivationsfördernd und ermögliche individuelle Lernwege und -tempi etc. (Döbeli Honegger 2016). Blicken wir auf den Einsatz digitaler Tools im Kontext Sport, wird deutlich, dass im Spitzensport die didaktischen Möglichkeiten beispielsweise das Videofeedback und das Veranschaulichungen von Techniken durch Videosequenzen genutzt werden, diese jedoch erst nach und nach im schulischen Sportunterricht ankommen. Beispiele dafür sind in den deutschen Praxis- und Themenheften für den Sportunterricht zu finden. ${ }^{4}$ Andererseits gehe von vom Medieneinsatz im Unterricht die Gefahr der Ablenkung aus, sie würden süchtig machen, seien Zeitfresser und ihnen fehle es an didaktischem Mehrwert (vgl. ebd., 175ff.).

Insgesamt wird der Vorbehalt, dass die Bewegungszeit der Schülerinnen und Schüler durch den Einsatz digitaler Medien zu kurz komme, häufig angebracht, doch gerade im Kontext des Sportunterrichts erhält dieser eine besondere Gewichtung. So besteht die Angst, dass sich Kinder durch die Nutzung digitaler Medien im Unterricht zu wenig bewegen und dies zu Haltungsschäden und Übergewicht führe (ebd., 36). Es gilt, diese Potenziale und Fallstricke im Zusammenhang mit praktischen Unterrichtsprojekten und -erfahrungen zu betrachten, zu analysieren und auszuloten.

\subsection{Das Lernen über Medien}

Diese weitere unterrichtliche Dimension wird in erster Linie von Seiten der Medienpädagogik relevant gesetzt. Um die Heranwachsenden zu einer selbstbestimmten, kritischen, sozial verantwortlichen und kreativen $\mathrm{Me}$ diennutzung zu befähigen, braucht es über fachdidaktische und fachliche Zielsetzungen des Medieneinsatzes hinaus auch medienpädagogische Ideen und Einflüsse für den Grundschulunterricht (Tulodziecki, Herzig, und Grafe 2019; Irion 2016a, 2016b). Hier sind beispielsweise das Wissen über rechtliche Grundlagen zu nennen und auch die Gestaltung eigener Medienprodukte.

4 u.a. Sportpraxis Sonderheft «Digitale Medien» (2019), Grundschule Sport «Digitale Medien» (2019). 
Dies kann gerade im Zusammenhang mit handlungsorientierter $\mathrm{Me}$ dienarbeit (Schorb 2008, 51f) ermöglicht werden, welche gleichzeitig die Chance bietet, Medien nicht nur zu rezipieren, sondern auch zu produzieren, also quasi hinter die Kulissen der Medienproduktion zu schauen. Dadurch können sich Gestaltungs- und Reflexionskompetenzen auf der Basis einer produktiven und aktiven Auseinandersetzung bereits im Grundschulalter entwickeln. Diese Ziele sollen jedoch nicht in Konkurrenz zu fachlichen Zielen stehen, sondern diese bestenfalls auf innovative Art und Weise erweitern, ergänzen oder integrieren.

Als ständige Begleiterin in Planungs- und Evaluationsphasen der praktischen Unterrichtserprobungen ist die jüngste Strategie der Kultusministerkonferenz (KMK) Bildung in der digitalen Welt (2017) zu nennen. Die dort beschriebenen sechs Kompetenzbereiche sollen verpflichtend in die Curricula aller Unterrichtsfächer jeder Schulart integriert werden. Sie benennen dabei lediglich die Kompetenzen, Fähigkeiten und Kenntnisse, die die Schülerinnen und Schüler am Ende ihrer Pflichtschulzeit erworben haben sollen. Eine konkrete Ausgestaltung wird von der Strategie nicht vorgenommen, dies kritisiert der Grundschulverband in der Stellungnahme zum KMK-Beschluss, indem unter anderem grundschulgerechte Kompetenzen für die digitale Welt gefordert werden (Grundschulverband 2018, 3).

Die Gesellschaft für Fachdidaktik (GFD) hat in einem Positionspapier (2018) vier Ansatzpunkte als Reaktion auf die Strategie der KMK entwickelt, die die Synergien zwischen fachlichen Lernen und dem Lernen mit und über Medien herausstellen. Im folgenden Abschnitt werden diese vier Ansatzpunkte mit Blick auf den Sportunterricht dargelegt (Greve et al. 2020a)

\section{- 〈Fachliche Kompetenzen digital fördern〉}

Hier stehen die Fragen im Fokus, wie digitale Medien als Werkzeuge zur Unterstützung der fachlichen Kompetenzentwicklung in den Unterricht integriert werden können, wie der Einsatz digitaler Medien das Lehren und Lernen verbessern kann und wie die Heranwachsenden fachliche Kompetenzen auf digitale Anwendungs- und Handlungsfelder übertragen können. Augenmerk liegt auf der kriteriengeleiteten Auswahl als auch auf der rezeptiven und produktiven Nutzung von $\mathrm{Me}$ dien als Werkzeuge für unterrichtliche Zwecke unter Berücksichtigung fachlicher Spezifika. Übertragen auf den Sportunterricht bedeutet dies 
beispielsweise, dass spezielle Apps wie Coaches Eye eingesetzt werden, um gemeinsam mit den Schülerinnen und Schülern die Möglichkeiten des Videofeedbacks für das Lernen von Bewegung, Spiel und Sport zu nutzen.

\section{- (Digitale Kompetenzen fachlich fördern〉}

Die von der KMK genannten Kompetenzen (2017) sollen anhand konkreter Unterrichtsgegenstände der Fächer gefördert werden. Für den Sportunterricht könnte das so aussehen, dass Schülerinnen und Schüler bei einer Stationsarbeit das Erstellen einer Audiodatei erlernen, indem sie einen Arbeitsauftrag für ihre Mitschülerinnen und Mitschüler über ihre selbst ausgedachten Bewegungsabläufe in einer Bewegungslandschaft verständlich verbalisieren und aufnehmen.

- (Fachliche digitale Kompetenzen über die KMK-Standards hinaus〉 Der dritte Bereich beschäftigt sich mit den Veränderungen fachlicher Gegenstände durch die Digitalisierung. Hier sollen Schülerinnen und Schüler mit weiteren digitalen fachlichen Kompetenzanforderungen vertraut gemacht werden, die über die genannten Bereiche der KMK Strategie (2017) hinausgehen. Für den Sportunterricht der Grundschule sind in der Praxis bisher keine Unterrichtskonzepte bekannt, welche diesen Ansatz verfolgen. Für den Sportunterricht höherer Klassenstufen wären jedoch Fragen nach veränderten Spielregeln durch Digitalisierung und deren Wirkung auf die jeweilige Sportart (z.B. aktuelle Debatte zum Videobeweis im Fussball) denkbar. Ein ähnlicher Themenstrang kann die Rolle von Schiedsrichter*innen sein, deren Entscheidungen durch den Einsatz digitaler Medien in der aktuellen Sportkultur häufig korrigiert werden.

- 〈Digitale personale Bildung im Fachunterricht fördern〉

Dieser Bereich umfasst die fachspezifische Reflexions- und Kritikfähigkeit über das Leben in einer digital vernetzten Welt. Solch eine Verbindung ist in dem unten aufgeführten Beispiel des Projekts MeInSport geschehen, indem Kinder im Grundschulsportunterricht einen ActionTrailer drehten und dafür Elemente der Filmsprache und die Wirkungsweise von Bildeinstellungen kennenlernten. Durch die Produktion eines Films ist die Klasse auch auf Fragen des Urheberrechts- und Persönlichkeitsrechts gestossen, die dann direkt an diesem Beispiel zur Anwendung kam. 


\subsection{Schulsport in der Grundschule}

In einem ganzheitlichen Verständnis von Schule als Lebenswelt rückt die Bewegung in doppelter Hinsicht in den Blick: als ein durchgängiges pädagogisches Prinzip von Schulentwicklung einerseits und als elementarer Beitrag zu einer umfassenden Bildung andererseits. Dass der Sportunterricht als ein unverzichtbares Element der schulischen Bildung und Erziehung angesehen wird, mit dem Ziel, die Schülerinnen und Schüler zu einem lebenslangen Sporttreiben zu motivieren, ist in den «Gemeinsamen Empfehlungen der Kultusministerkonferenz und des Deutschen Olympischen Sportbundes zur Weiterentwicklung des Schulsports» (Kultusministerkonferenz und Deutschen Olympischen Sportbundes 2007) verankert.

«Der Sportunterricht ist so auszurichten, dass die Schüler*innen individuell gefordert und gefördert und zum außerunterrichtlichen Sporttreiben motiviert werden» (ebd., 5).

Betrachtet man die aktuellen Diskurs- und Begründungslinien des Schulsports, münden diese im so genannten Doppelauftrag (Prohl 2006), der so oder in ähnlicher Form seit der Jahrtausendwende in den bundesdeutschen Lehrplänen verankert ist: Zum einen die Erschliessung der Bewegungs-, Spiel- und Sportkultur und zum anderen die Entwicklungsförderung durch Bewegung, Spiel und Sport. Diese beiden Zielperspektiven führen zwei durchaus konträre fachdidaktische Strömungen der vergangenen vier Jahrzehnte zusammen: die pragmatisch-qualifikatorische Strömung und die kritisch-emanzipatorische Strömung. In der ersteren stellt das Sportartenkonzept (Söll 1995) die sport(art)-spezifische Vermittlung von Fähigkeiten und Fertigkeiten in den Mittelpunkt des Sportunterrichts. Der Bildungs- und Erziehungsauftrag besteht aus der Körperbildung (Förderung und Verbesserung der körperlichen Fähigkeiten und Fertigkeiten) und der Bewegungsbildung (Einführung in die Bewegungskultur). Dieses Konzept zeichnet sich durch ein enges Sportverständnis aus, welches auf der methodischen Ebene ein eher geschlossenes, deduktives Vorgehen bevorzugt. Aus der Kritik an dieser stark an der Sachstruktur des Sports orientierten Didaktik entwickelten sich in den späten 1970er Jahren kritischemanzipatorische Ansätze. In diesen fachdidaktischen Konzepten (bspw. das Konzept der Körpererfahrung nach (Funke-Wieneke 2001) stehen die 
Schülerinnen und Schüler im Mittelpunkt. Funke-Wieneke sieht die Befähigung der Lernenden zum Umgang mit dem eigenen Körper, zur aktiven Auseinandersetzung mit den individuellen Bewegungsbedürfnissen und zum Erfahren vielfältiger Zugänge zur Bewegungs-, Spiel- und Sportkultur als zentral im Sportunterricht. Bewegung wird als Medium der aktiven Auseinandersetzung mit der Umwelt interpretiert, die Persönlichkeitsund Identitätsentwicklung stellen die zentralen Leitziele dar. Sportunterricht soll am Interessen- und Fragehorizont der Schülerinnen und Schüler orientiert sein und Raum für selbsttätiges, experimentierfreudiges, erkundendes, entdeckendes und problemlösendes Handeln bieten.

Eine vermittelnde Funktion zwischen diesen beiden divergierenden Konzepten erfüllt das Konzept der Handlungsfähigkeit (Kurz 2000). In dieser Denkfigur sollen Schülerinnen und Schüler zum selbsttätigen Handeln im Sport qualifiziert werden. Der Sport wird als sinngeleitetes und sinnsuchendes Handeln interpretiert und kann auf unterschiedliche Weise als sinnvoll erfahren bzw. im Sinn belegt werden. Daraus erwächst das Unterrichtsprinzip der Mehrperspektivität, welches den Schülerinnen und Schüler die Vielfalt des sportlichen Handelns entlang der sechs pädagogischen Perspektiven eröffnet (Leistung, Wagnis, Kooperation, Gesundheit, Eindruck, Ausdruck). Handlungsfähig im Sport sind nach Kurz jene, die die vorgefundenen Angebote des Sports kritisch auf ihre Sinnhaftigkeit prüfen (können) und aus der Vielfalt sportlicher Sinnbezüge begründet auswählen und diese im eigenen Sporttreiben befriedigend und lebensbereichernd verwirklichen können. Das Prinzip der Mehrperspektivität nach Kurz ist für die aktuelle sportdidaktische Fachdiskussion in jeder Hinsicht als äusserst prägend anzusehen.

Prohl hat anknüpfend an die beschriebene Diskussion den Terminus des Doppelauftrags des Erziehenden Sportunterrichts eingeführt (2006). Er stellt in einer bildungstheoretischen Rahmung die Frage, inwieweit der Sportunterricht zur Allgemeinbildung beitragen kann, ohne dabei die Bewegungskultur resp. den Sport zu vernachlässigen (Prohl 2006, 198). Der Sportunterricht dient somit der Sachaneignung (im Sinne des Erwerbs sportlicher Kompetenzen) und im Vollzug der Sachaneignung dem Erwerb von Schlüsselkompetenzen allgemeiner Bildung (im Sinne der Persönlichkeitsentwicklung). Zusammenfassend spricht Prohl von der 
«Bewegungsbildung als qualitativ strukturiertem Erfahrungsprozess» (ebd., 175), was bedeutet, dass Bewegungsbildung (als Sachaneignung, wobei die Sache der Sport ist) in einem Sportunterricht nach den Grundsätzen des Doppelauftrags im Lichte der Allgemeinbildung stattfindet. Die dargelegte Begründungslinie des Doppelauftrags findet sich in den Lehrplänen der Länder wieder (Ruin und Stibbe 2016). Im Rückgriff auf Kurz spiegeln die sechs pädagogischen Perspektiven den Gegenwarts- und Zukunftsbezug wieder:

- Wahrnehmungsfähigkeit verbessern, Bewegungserfahrungen erweitern;

- Sich körperlich ausdrücken, Bewegung gestalten;

- Etwas wagen und verantworten;

- Das Leisten erfahren, verstehen und einschätzen;

- Kooperieren, wettkämpfen, sich verständigen;

- Gesundheit fördern, Gesundheitsbewusstsein entwickeln.

In der aktuellsten Lesart eines mehrperspektivischen Unterrichts plädieren Balz und Neumann (2015) für einen flexiblen Umgang mit diesen Perspektiven und verweisen angesichts der wachsenden Heterogenität der Schülerinnen und Schüler auf eine mögliche Veränderung der Anzahl, Benennung und Ausgestaltung.

Die Inhalte des Sportunterrichts sind in den aktuellen Lehrplänen der Länder zumeist in Form von Bewegungsfeldern aufgeführt, mit dem Ziel einer Erweiterung und Öffnung der Inhalte über die traditionellen Sportarten hinaus (Stibbe 2011). Dieses erweiterte Sport- und Bewegungsverständnis eröffnet die Orientierung an den Bedürfnissen und Interessen der Lernenden. Stibbe (2000) fasst den Wandel der Lehrpläne prägnant zusammen: «Von einem instruktionsorientierten Sportunterricht, der auch erzieht, zu einem erziehenden Sportunterricht, der auch instruiert.»

Der Sportunterricht wird bezüglich seiner Inhalte und Inszenierungsoptionen auch von gesellschaftlichen Veränderungen tangiert. Der Begriff der Inszenierung findet in der sportdidaktischen Diskussion zumeist im Sinne von Dietrich und Landau (1990) Verwendung. Dieser Interpretation wird auch an dieser Stelle gefolgt. Die Nutzung des Begriffs erfolgt angelehnt an das Sprachjargon der Theaterwelt, Schülerinnen und Schüler 
sowie die Lehrkraft füllen im Kontext des Sportunterrichts verschiedene Rollen aus und konstruieren das Unterrichtsgeschehen gemeinsam durch die Ausgestaltung dieser verschiedenen Rollen. Dieses Rollenspiel, in Anlehnung an die Tradition des Symbolischen Interaktionismus nach Mead (1986), ist für Unterrichtssituationen im Sportunterricht, die durch emotional-ästhetisch aufgeladene Handlungen der Akteurinnen und Akteure gekennzeichnet ist, genuin.

Zu den Veränderungen zählt die Omnipräsenz digitaler Medien im Alltag von Kindern und Jugendlichen, auf institutioneller Ebene die digitale Ausstattung der Schullandschaft und auf der bildungspolitischen Ebene die Digitalisierungsoffensive (Bsp. Digitalpakt und andere landes- und bundesweite Förderlinien). Herauszustellen ist, dass bzgl. des Einsatzes von digitalen Medien im Sportunterricht bisher kaum Forschungsergebnisse vorliegen, unabhängig ob im Bereich der Grundschule oder in anderen Schulformen. Es liegt somit ein Desiderat vor (Greve et al. 2020b; Wendeborn 2019). Angesichts der gesellschaftlichen Rahmenbedingungen und den oben ausgeführten fachdidaktischen Überlegungen möchten wir im Folgenden die theoretische Anschlussfähigkeit an medienpädagogische Zielperspektiven auf der Basis durchgeführter Unterrichtsforschung entfalten und diskutieren.

\section{Medienpädagogik im Sportunterricht: das Projekt MeIn Sport}

«MeIn Sport - Medienpädagogik und Inklusion im Sportunterricht der Grundschule» ${ }^{5}$ ist im Anschluss an das Projekt «Herausforderungen in der Grundschule digital begegnen ${ }^{6}$, in Kooperation der Universität Hamburg mit der Leuphana Universität Lüneburg entstanden. Im Fokus des Projekts steht die gemeinsam vorgenommene Entwicklung von Konzepten und konkreten Unterrichtsideen aus Sportdidaktik und Medienpädagogik,

5 Das Projekt wurde als Kleinforschungsprojekt an der Leuphana gefördert. Infolgedessen ist im Rahmen des durch die Qualitätsoffensive Lehrerbildung geförderten Projektstrangs 'codib' (Competences for Digital-Enhanced Individualized Practice) an der Leuphana eine Fortsetzung der Forschungsaktivitäten zu digitalen Medien im Sportunterricht eingeworben worden.

6 Gefördert von der Deutschen Telekom Stiftung. 
welche die Möglichkeiten digitaler Medien produktiv aufgreifen und in fachdidaktisch begründbare und für die Altersgruppe und ihrer Heterogenität angemessene Unterrichtsvorhaben übersetzen. Dabei wurde eng mit den Sportlehrkräften der Klassen zusammengearbeitet.

Die Evaluation der Unterrichtseinheiten erfolgte anhand qualitativer Forschungsmethoden, neben Videographie und teilnehmender Beobachtung kamen insbesondere leitfadengestützte Interviews mit den Schülerinnen und Schülern sowie den Lehrkräften zum Einsatz, in denen die Beteiligten u.a. zu den unterrichtlichen Prozessen und Arbeitsprodukten befragt werden. Die Daten werden im Moment mithilfe der Kodierverfahren der Grounded Theory (Strauss und Corbin 1999; Krieger 2016) ausgewertet. Bisher liegen lediglich für die Kinderinterviews erste Ergebnisse vor, sodass die datengestützte und systematische Evaluation nicht Teil dieses Beitrags ist.

In dem Projekt sind bisher vier Unterrichtseinheiten in ersten und zweiten Klassen entstanden, die im Folgenden genauer beschrieben werden.

Der Einsatz von Tablets ermöglicht in den im folgenden vorgestellten Beispielen eine Neugestaltung (3.1 und 3.3) beziehungsweise ein Neudenken (3.2 und 3.4) von Optionen der Aufgabenstellung und Lernanlässe. Mit Verweis auf das SAMR-Modell (Puentedura 2006) wird hier die Ebene der Transformation (Modifikation und Neubestimmung) erreicht.

\subsection{Zirkus im Sportunterricht - Das Tablet als Eintrittskarte}

In der Unterrichtseinheit /Zirkus im Sportunterricht - Das Tablet als Eintrittskarte〉 sollen Schülerinnen und Schüler dem Clown Krusty helfen, sich neue Kunststücke für seine Zirkuskür zu überlegen. Krusty meldet sich zu Beginn der Unterrichtseinheit über E-Mail und Videobotschaft bei den Kindern und bittet diese um Hilfe. In der Sporthalle sind vier bis fünf Stationen (Hula-Hoop, Jonglage, Zirkusgeräte, Akrobatik) aufgebaut, an denen je ein Tablet liegt. Darauf sind Beispielbilder und Videos zu sehen, die Anregungen für die einzelnen Stationen geben. An der Jonglage-Station sind beispielsweise Videos der Lehrkraft zu sehen, die mit verschiedenen Materialien (z.B. Tücher oder Bälle) und in verschiedenen Schwierigkeitsstufen 
(z.B. ein Ball, zwei Bälle, drei Bälle) jongliert. Die Aufnahmen sind in Zeitlupe gemacht, um Bewegungsabläufe gut nachverfolgen zu können. Die Schülerinnen und Schüler können sich mit den Tablets aufnehmen ${ }^{7}$ und so ihre Ideen an Krusty weitergeben. Zu Beginn jeder Stunde gibt es ein Feedback von Krusty zu bereits aufgenommenem Material. In der letzten Stunde der Einheit präsentieren die Schülerinnen und Schüler Krusty eine kleine Kür mit neuen Kunststücken für seinen Zirkus. Hier können die Schülerinnen und Schüler sich aussuchen, welche Aufnahme sie zeigen wollen und so der körperlichen Exponiertheit (Krieger und Veit 2019) entgehen, die beim einmaligen präsentieren entstehen kann.

\section{2 ...und Action!}

Im Sportunterricht produzieren Kinder der zweiten Klasse einen bewegenden Trailer, der mit einem Tablet in einer Bewegungslandschaft aufgenommen wird (Thumel et al. 2019) ${ }^{8}$. Nachdem die Schülerinnen und Schüler die vorbereitete Bewegungslandschaft erkundet haben, bekommen sie von der Lehrkraft eine Einführung in das Thema Perspektiven (Frosch-, Vogel- und Normalperspektive). Die Kinder erarbeiten sich in der ersten Stunde die Wirkung dieser Perspektiven anhand eigener kurzer Videoaufnahmen. Im Sitzkreis wird anschliessend geklärt, was ein Trailer ist und welche Funktion dieser hat. Um einen Trailer zu produzieren, lernen die Kinder die Einstellungsgrössen (Totale, Halbnah, Nah, Detailaufnahme) kennen. Diese werden anhand der Symbolbilder, die in der App angezeigt werden, erläutert. Zusätzlich zu dieser mündlichen Erklärung liegen den Kindern auf ihren Tablets filmische Erläuterungen der Einstellungsgrössen vor. Das hilft den Kindern, sich wiederholt mit den Begrifflichkeiten vertraut zu machen und gibt Kindern, welche die Erklärung der Lehrkraft nicht verstehen konnten, die Möglichkeit sich diese noch einmal anzuschauen. Zusätzlich können Bilder vergrössert, sowie Videos gestoppt und wiederholt angeschaut werden. In einer kurzen Einführung in die Software wird gezeigt, wie die Kinder eine Szene aufnehmen und den Ausschnitt

7 In dieser und in den folgenden zwei Unterrichtsbeispielen stand es den Schülerinnen und Schülern frei sich filmen zu lassen. Dieses Persönlichkeitsrecht wurde im Unterricht thematisiert.

8 Es wurde dir iOS App iMovie verwendet. 
auswählen. Dann haben die Kinder bis zur letzten Stunde Zeit ihre Aufnahmen zu machen und so ihren Trailer zu vervollständigen. Die Aufgabe war es sich spannende Bewegungen an unterschiedlichen Geräten zu überlegen, diese zu üben, sie aufzunehmen und im letzten Schritt die besten Sequenzen für den Trailer auszuwählen. In der letzten Stunde wird der Trailer gemeinsam im Klassenzimmer angeschaut und besprochen.

\subsection{Fussball Kunststücke}

In dieser Unterrichtseinheit eignen sich die Schülerinnen und Schüler mit Hilfe von Tablets verschiedene Fussballtricks an und präsentieren diese. Die Aufgabe dafür kommt in einer E-Mail von einem berühmten Fussballstar. In der Sporthalle verteilt stehen verschiedene Stationen bestückt mit Kegeln und Hütchen, kleinen Toren, Kästen und Matten. An jeder Station liegt ein Tablet mit Ideen und Beispielvideos in Slow-motion. Die Kinder denken sich neue Tricks aus oder ahmen die Videobeispiele nach. Die geübten Kunststücke werden von den Mitschülerinnen und Mitschülern gefilmt. Abschliessend reflektierten die Kinder in Kleingruppen die Schwierigkeitsgrade der Kunststücke und die Wirkung ihrer Aufnahmen.

\subsection{Dschungel-Bewegungslandschaft}

In dieser Unterrichtseinheit geht es um den tabletgestützten Aufbau einer Dschungel-Bewegungslandschaft (Greve et al. 2019). Auf den Tablets ist in einem Video ein Plüschaffe ${ }^{10}$ zu sehen, der den Kindern den Aufbau der verschiedenen Stationen erklärt und visualisiert. Zudem beschreiben animierte Sportgeräte die Regeln des sicheren Aufbaus in der Sporthalle (z.B. Matte: «Mich trägt man nur zu viert!»). Durch die Videos haben die Kinder die Möglichkeit sich weiderholt den Ablauf des Geräteaufbaus anzuschauen. In der Mitte der Halle liegt ein weiteres Tablet. Dieses «Hilfetablet» nutzt die Talkerapp LetMeTalk. Mit dieser App können die Geräte in der Halle abfotografiert werden. Tippt man nun auf die Geräte wird deren Name laut und deutlich vorgelesen, sodass sich die Kinder im Unterricht

9 Eine kritische Auseinandersetzung mit dieser Unterrichtseinheit findet sich in Greve (2019).

10 Der Affe wurde mit Hilfe der App Chatterpix animiert. 
jederzeit informieren können. Nach dem Aufbau, fängt die Bewegungszeit an. Die Aufgabenstellung finden sich als Clips auf den Tablets. Wie in Kapitel 2.2 erwähnt, sprechen die Kinder selbst erdachte Bewegungen als Aufgaben für ihre Mitschülerinnen und Mitschüler auf, welche im Anschluss angehört und ausprobiert werden.

\section{Inszenierung von Sportunterricht durch den Einsatz digitaler Medien}

Das gemeinsame Inszenieren von Unterricht durch Schülerinnen und Schüler und wandelt sich durch den Einsatz digitaler Medien in verschiedenen Komponenten. Die Kommunikation der Lehrkraft mit den Schülerinnen und Schülern sowie die Kommunikation der Schülerinnen und Schüler untereinander erfährt durch den Einsatz digitaler Medien Veränderungen: Die Kinder erhielten, anstatt einer rein verbalen Anweisung der Lehrkraft, die Möglichkeit, die Aufbauanleitung audiovisuell unterstützt aufbereitet zu erhalten. Dies entlastet einerseits die Lehrkraft und andererseits wurde durch den Einsatz der Tablets verschiedene Lernkanäle angesprochen, womit der heterogenen Struktur der Lerngruppe im Anfangsunterricht Rechnung getragen werden kann. Wie Medien dahingehend gestaltet sein sollten, um das Lernen zu fördern und Barrieren zu umgehen, kann mittlerweile gut begründet werden (Herzig 2014): es ist ein höherer Lernerfolg zu erwarten, wenn Informationen als Bild und Text präsentiert werden (Fletcher und Tobias 2005) oder Bezüge zwischen Bild, Ton und Text hergestellt werden werden (Schneider et al. 2018)

Der Einsatz von mit Sprache unterlegten Fotos zum Aufbau einer Gerätelandschaft (vgl. 3.4) kann hier als anschauliches Beispiel dienen. Durch diese Möglichkeit des mehrmaligen Anschauens der Anleitung und die Option der Diskussion der Anleitung mit den Mitschülerinnen und Mitschüler anhand der Bilder auf dem Tablet eröffnen sich digitale Hilfestellungen für die Kinder, sie konnten den Aufbau selbstständiger aufbauen.

Da die Lehrkraft den Aufbau nicht anleiten musste, konnte sie die Gruppen gezielt beim Aufbau ihrer Stationen unterstützen, die dabei Hilfe benötigten. 
Die Schülerinnen und Schüler hatten beim Erkunden der Bewegungslandschaft die Möglichkeit, Mitschülerinnen und Mitschüler zu filmen bzw. gefilmt zu werden und somit ihre Bewegungen an den einzelnen Stationen mithilfe der Videos anzuschauen, und ggf. Bewegungsabläufe zu reflektieren und Korrekturen vornehmen. Ausserdem bestand die Möglichkeit des Aufsprechens von Aufgaben und dadurch des Weitergebens von Ideen. Die Möglichkeit des Konservierens von Bewegungen bzw. Ideen für Bewegungen ist als neue Option anzusehen, welche durch den Einsatz der Tablets entsteht.

Der flüchtige Moment der Bewegung kann durch das Aufnehmen kurzzeitig konserviert werden und z.B. später im Klassenraum über Beamer angeschaut werden. Durch die Mobilität der Aufnahmen bzw. der Konservierung der Bewegungen entsteht eine Öffnung des Raumes. Die Aktivitäten in der Sporthalle können zeitversetzt in den Klassenraum geholt werden. Es entstehen Möglichkeiten der Reflexion und der Wertschätzung von Handlungen im Sportunterricht, die ohne den Einsatz der digitalen Medien nicht möglich wären. Der abgeschottete Raum der Sporthalle wird aufgelöst, sodass auch fächerübergreifende Aspekte in den Fokus rücken können. Bspw. kann ein Trailer, in dem Bewegungen mit Musik unterlegt sind, auch im Musikunterricht Thema sein.

Der Kerninhalt des Sportunterrichts, Bewegung, Spiel und Sport, kann durch den Einsatz digitaler Medien befördert werden. Oftmals wird der Einsatz digitaler Medien mit einer Verringerung der Bewegungszeit gleichgesetzt. Dem entgegen kann generell kann festgehalten werden, dass das Lösen von Aufgaben im Sportunterricht durch den Vollzug von Bewegungen passiert. Dies kann nicht durch den Einsatz von Tablets substituiert werden. Das Tablet kann nur bei der Inszenierung von Sportunterricht helfen, diesen aber nicht ersetzen. Auch die Inszenierung wird immer von den Lehrkräften getragen, und ist abhängig von am Inhalt zielgerichtet ausgewählten Methoden und Lernzielen. Hier können digitale Medien u.a. eine unterstützende Funktion für den Fachunterricht in der Grundschule haben. In den angeführten Beispielen konnte gezeigt werden, dass die digital gestützte Inszenierung von Sportunterricht in der Grundschule, die in der Praxis häufig mit Rahmengeschichten erfolgte, durch den Einsatz digitaler Medien intensiviert wurde (vgl. 3.4: das Kuscheltier kann mit 
Hilfe der Videobearbeitung sprechen). Die Kinder tauchen ab in eine fiktive Welt, lösten dort Aufgaben, wurden herausgefordert und hatten durch die motivierende und anregende Inszenierung besondere Bewegungserlebnisse. Diese Form kann gerade für Kinder, die eher weniger Bezug zu sportlicher Betätigung aufweisen, besonders wichtig sein.

An dieser Stelle soll realistisch betont werden, dass die Entwicklung und konkrete Planung solcher Unterrichtsvorhaben herausfordernd ist. Dies gelang im vorgestellten Projekt durch die Kooperation von Medienpädagogik und Sportdidaktik, genauso wie in der Schule Sportlehrkräfte im Regelfall auf die Unterstützung der Medienbeauftragten angewiesen sind. Das Projekt, welches explorativen Charakter aufweist, möchte innovative Anregungen für den Unterricht in der Grundschule liefern. Dazu ist die Evaluation des Projekts als Start für weitere Forschungsvorhaben in diesem Feld gedacht.

\section{Literatur}

«SportPraxis», Redaktion. 2019. Digitale Medien im Sportunterricht. SportPraxis. Sonderheft.

Aßmann, Sandra. 2015. "Entgrenzung von Schule in der digitalen Welt.» Computer + Unterricht Seelze: Friedrich Verlag (99): 10-13.

Balz, Eckart, und Peter Neumann. 2015. «Mehrperspektivischer Sportunterricht.» Sportpädagogik $39(3+4): 2-7$.

Bastian, Jasmin. 2017. «Lernen mit Medien- Lernen über Medien? Eine Bestandsaufnahme zu aktuellen Schwerpunktsetzungen.»DDS- Die Deutsche Schule 109 (2): 146-62. https://www.waxmann.com/index.php?eID=download\&id_ artikel=ART102179\&uid=frei.

Dietrich, Knut, und Gerhard Landau. 1990. Sportpädagogik: Grundlagen, Positionen, Tendenzen. Rororo Sport 8623. Reinbeck: Rowohlt.

Döbeli Honegger, Beat. 2016. Mehr als O und I Schule in einer digitalisierten Welt. Bern: hep verlag.

Dräger, Jörg und Ralph Müller-Eiselt. 2018. Die digitale Bildungsrevolution: Der radikale Wandel des Lernens und wie wir ihn gestalten können. München: Deutsche Verlags-Anstalt.

Eickelmann, Birgit und Mario Vennemann. 2014. «Nutzung digitaler Medien im naturwissenschaftlichen Unterricht der Grundschule.» In Grundschule in der digitalen Gesellschaft: Befunde aus den Schulleistungsstudien IGLU und TIMSS 2011, hrsg. von Birgit Eickelmann, Ramona Lorenz, Mario Vennemann, Julia Gerick und Wilfried Bos. 1. Aufl., 73-84. Münster: Waxmann. 
Eickelmann, Birgit, Ramona Lorenz, Mario Vennemann, Julia Gerick, und Wilfried Bos, Hrsg. 2014. Grundschule in der digitalen Gesellschaft: Befunde aus den Schulleistungsstudien IGLU und TIMSS 2011. Münster, Westf: Waxmann.

Fletcher, J. d. und Sigmund Tobias. 2005. «The Multimedia Principle.»In The Cambridge handbook of multimedia learning, hrsg. von Richard E. Mayer, 117-34. Cambridge, U.K., New York: Cambridge University Press.

Funke-Wieneke, Jürgen. 2001. «Was ist zeitgemäßer Sportunterricht?». Sportpädagogik 25 (1): 47-51.

Gesellschaft für Fachdidaktik e.V. 2018. «Fachliche Bildung in der digitalen Welt: Positionspapier der Gesellschaft für Fachdidaktik.» Zugriff am 20. Dezember 2019. https://www.fachdidaktik.org/wordpress/wp-content/uploads/2018/07/ GFD-Positionspapier-Fachliche-Bildung-in-der-digitalen-Welt-2018-FINALHP-Version.pdf.

Greve, Steffen, Mareike Thumel, Florian Jastrow, Claus Krieger, und Jessica Süßenbach. 2019. «Eine Dschungel-Bewegungslandschaft mithilfe von Tablets inszenieren.» Sportpraxis.

Greve, Steffen, Mareike Thumel, Florian Jastrow, Claus Krieger, und Jessica Süßenbach. 2020a. «Digitale Medien im Sportunterricht der Grundschule Ein Update für die Sportdidaktik?!». In Digitale Bildung im Grundschulalter: Grundsatzfragen zum Primat des Pädagogischen, hrsg. von Mareike Thumel, Rudolf Kammerl und Thomas Irion, o.A.

Greve, Steffen, Mareike Thumel, Florian Jastrow, Anja Schwedler, Claus Krieger, und Jessica Süßenbach. 2020b. «Digitale Medien im Sportunterricht- Mehrwerte und Herausforderungen interdisziplinärer Verzahnung.» Sportunterricht 69: o.A.

Grundschulverband. 2018. «Digitale Mündigkeit beginnt in der Grundschule! Stellungnahme des Grundschulverbands zum «DigitalPakt Schule» und zum KMK-Beschluss «Bildung in der digitalen Welt».».

Heinzel, Friederike, und Katja Koch, Hrsg. 2017. Individualisierung im Grundschulunterricht. Wiesbaden: Springer Fachmedien Wiesbaden.

Herzig, Bardo. 2014. Wie wirksam sind digitale Medien im Unterricht? Bertelsmann Stiftung.

Herzig, Bardo und Sandra Aßmann. 2014. «Entgrenzung Von Schule in Der Digitalen Welt.» In Schoolss Out? Informelle Und Formelle Medienbildung, hrsg. von Sandra Aßmann, Dorothee M. Meister, Anja Pielsticker und Gesellschaft für Medienpädagogik und Kommunikationskultur in der Bundesrepublik Deutschland, 43-55. Schriften zu Medienpädagogik 48. München: kopaed.

Hüther, Jürgen, und Bernd Schorb, Hrsg. 2005. Grundbegriffe Medienpädagogik. 4., vollst. neu konzipierte Aufl. München: kopaed.

Irion, Thomas. 2016a. Digitale Medienbildung in der Grundschule - Digitale Kids, quietschende Tafeln, staubige Mäuse und wischende Finger. Ringvorlesung. 
Irion, Thomas. 2016b. «Digitale Medienbildung in der Grundschule. Primarstufenspezifische und medienpädagogische Anforderungen.»In Neue Medien in der Grundschule 2.0 Grundlagen - Konzepte - Perspektiven, hrsg. von Markus Peschel, Thomas Irion und Arbeitskreis Grundschule, 16-32. Beiträge zur Reform der Grundschule Band 141. Frankfurt am Main: Grundschulverband e.V.

Jörissen, Benjamin, und Stephan Münte-Goussar. 2015. "Medienbildung als Schulentwicklung. Oder: Wie man ein Trojanisches Pferd zähmt.» Computer + Unterricht (Heft 99): S. 4-9.

Junge, Thorsten und Horst Niesyto. 2019. Digitale Medien in der Grundschullehrerbildung: Erfahrungen aus dem Projekt dileg-SL. Medienpädagogik interdisziplinär.

Kahlert, Joachim, Kai Nitsche, und Klaus Zierer, Hrsg. 2013. Räume zum Lernen und Lehren: Perspektiven einer zeitgemäßen Schulraumgestaltung. Bad Heilbrunn: Verlag Julius Klinkhardt.

Kammerl, Rudolf. 2018. "Bildung und Lehrerbildung im digitalen Wandel. Zur Forderung nach einem (Primat des Pädagogischen).» In Medienpädagogik: Herausforderungen für Lernen und Bildung im Medienzeitalter, hrsg. von Theo Hug, 19-32. Innsbruck: innsbruck university press.

Krauthausen, Günter, Kerstin Michalik, Claus Krieger, Florian Jastrow, Christina Metzler, Alexandra Pilgrim, Anja Schwedler, und Mareike Thumel, Hrsg. 2020. Tablets im Grundschulunterricht: Fachliches Lernen, Medienpädagogik und informatorische Bildung. Baltmannsweiler: Schneider Verlag Hohengehren GmbH.

Krieger, Claus. 2016. «Pragmatische Verwendung der Kodierverfahren der Grounded Theory - und darüber hinaus?». In Bildungsforschung revisited, hrsg. von Gregor Lang-Wojtasik und Stefan König. 1. Auflage, 43-59. Weingartner Dialog für Forschung 1. Ulm: Klemm + Oelschläger.

Krieger, Claus, und Janes Veit. 2019. «Digitale Medien im Sportunterricht.» https://wimasu.de/digitalemedien.

Kultusministerkonferenz. 2019. "Standards für die Lehrerbildung: Bildungswissenschaften: (Beschluss der Kultusministerkonferenz vom 16.12.2004 i. d. F. vom 16.05.2019).» https://www.kmk.org/fileadmin/veroeffentlichungen_beschluesse/2004/2004_12_16-Standards-Lehrerbildung-Bildungswissenschaften.pdf.

Kultusministerkonferenz, und Deutscher Olympischer Sportbund. 2007. «Gemeinsame Handlungsempfehlungen der Kultusministerkonferenz und des Deutschen Olympischen Sportbundeszur Weiterentwicklung des Schulsports.». https://www.bildung-mv.de/export/sites/bildungsserver/downloads/ KMK-Handlungsempfehlungen.pdf.

Kultusministerkonferenz KMK. 2012. Medienbildung in der Schule, Nr. 21.10.2015. http://www.kmk.org/fileadmin/veroeffentlichungen_beschluesse/2012/2012_03_08_Medienbildung.pdf. 
Kurz, Dietrich. 2000. «Pädagogische Grundlagen des Schulsports in NordrheinWestfalen.» In Erziehender Schulsport: Pädagogische Grundlagen der Curriculumrevision in Nordrhein-Westfalen. 1. Aufl., 9-55. Curriculumentwicklung NRW. Bönen: Verl. für Schule und Weiterbildung Dr.-Verl. Kettler.

Mead, George Herbert. 1986. Geist, Identität und Gesellschaft. Frankfurt am Main: Suhrkamp.

Mpfs. Medienpädagogischer Forschungsverbund Südwest. 2019. KIM-Studie 2018. Kindheit, Internet, Medien. Stuttgart. https://www.mpfs.de/fileadmin/files/ Studien/KIM/2018/KIM-Studie_2018_web.pdf.

Petko, Dominik, Beat Döbeli Honegger, und Doreen Prasse. 2018. «Transformation in Bildung und Schule: Facetten, Entwicklungs linien und Herausforderungen für die Lehrerinnen- und Lehrerbildung.» Beiträge zur Lehrerinnen- und Lehrerbildung 26 (2): 157-74. https://bzl-online.ch/download/271/BzL_182_157174Petkoetal.pdf.

Prohl, Robert. 2006. Grundriss der Sportpädagogik. 2., stark überarb. Aufl. Wiebelsheim: Limpert.

Puentedura, Ruben R. 2006. "Transformation, Technology, and Education in the state of Maine.». http://www.hippasus.com/resources/tte/.

Ruin, Sebastian und Günter Stibbe. 2016. «Erziehender Sportunterricht und kompetenzorientierte Lehrpläne.» In Didaktik des Schulsports: Beiträge zu einer zeitgemäßen Diskussion, hrsg. von Günter Stibbe und Martin Holzweg. Nachdruck von einzelnen, teilweise überarbeiteten Beiträgen zur Didaktik des Schulsports aus der Zeitschrift Sportunterricht aus den Jahren 1983 bis 2016, 147-53. Sportunterricht Sonderheft. Schorndorf: hofmann.

Rummler, Klaus, Beat Döbeli Honegger, Heinz Moser, und Horst Niesyto, Hrsg. 2016. Medienbildung und informatische Bildung - quo vadis? Bd. 25. Themenheft. Zürich. https://doi.org/10.21240/mpaed/25.X.

Schorb, Bernd. 2008. «Handlungsorientierte Medienpädagogik.»In Handbuch Medienpädagogik, hrsg. von Uwe Sander, Friederike von Gross und Kai-Uwe Hugger. 1. Aufl., 51-60. Wiesbaden: VS Verlag für Sozialwissenschaften.

Schneider, S., Maik Beege, Steve Nebel und G. D. Rey. 2018. A Meta-analysis of how Signaling Affects Learning with Media. Elsevier B.V.

Söll, Wolfgang. 1995. «Sportunterricht ohne Sportarten.» Körpererziehung 45 (10): $323-28$.

Stibbe, Günter. 2000. «Vom Sportartenprogramm zum erziehenden Sportunterricht. Zur curricularen Neubesinnung über den Schulsport in NordrheinWestfalen.» Sportunterricht 49 (7): 212-19.

Stibbe, Günter. 2011. «Standards, Kompetenzen und Lehrpläne im Fach Sport - Einführung.»In Standards, Kompetenzen und Lehrpläne: Beiträge zur Qualitätsentwicklung im Sportunterricht, hrsg. von Günter Stibbe und Heinz Aschebrock, 11-15. Reihe Sport 16. Schorndorf: hofmann.

Strauss, Anselm L., und Juliet M. Corbin. 1999. Grounded TheorY: Grundlagen Qualitativer Sozialforschung. Weinheim: Beltz, Psychologie Verl.-Union. 
Thom, Sabrina, Julia Behrens, Ulrich Schmid, und Lutz Goertz. 2017. «Monitor Digitale Bildung. Digitales Lernen an Grundschulen.» https://www.bertelsmann-stiftung.de/fileadmin/files/BSt/Publikationen/GrauePublikationen/ BST_DigiMonitor_Grundschulen.pdf.

Thumel, Mareike, Florian Jastrow, Steffen Greve, Claus Krieger, und Jessica Süßenbach. 2019. «... und Action! Bewegungsfördernder Einsatz von Tablets.» Grundschule Sport (23): 6-9.

Tillmann, Alexander, und Claudia Bremer. 2017. «Einsatz von Tablets in Grundschulen: Umsetzung und Ergebnisse des Projekts Mobiles Lernen in Hessen (MOLE).» In Tablets in Schule und Unterricht Forschungsmethoden und -perspektiven zum Einsatz digitaler Medien, 241-76. Wiesbaden: Springer VS.

Tulodziecki, Gerhard, Bardo Herzig, und Silke Grafe. 2019. Medienbildung in Schule und Unterricht: Grundlagen und Beispiele. 2., vollständig überarbeitete und aktualisierte Auflage. Utb 3414. Bad Heilbrunn: Verlag Julius Klinkhardt.

Wendeborn, Thomas. 2019. «Wer nicht mit der Zeit geht, geht mit der Zeit - Zum Sportunterricht in einer digital revolutionierten Gesellschaft.» Leipziger Sportwissenschaftliche Beiträge (2): 9-25.

Zimmermann, Ann Susann. 2017. «Lernen mit Tablets in der Grundschule. Eine qualitative Studie zur Erfassung der kindlichen Perspektive.»In Individualisierung im Grundschulunterricht, hrsg. von Friederike Heinzel und Katja Koch, 142-46. Wiesbaden: Springer Fachmedien Wiesbaden. 